\title{
Robust State Estimation and Fault Detection Combining Unknown Input Observer and Set-membership Approach
}

\author{
Feng $\mathrm{Xu}^{1}$, Junbo Tan ${ }^{1}$, Xueqian Wang ${ }^{1, *}$, Vicenç Puig ${ }^{2}$, Houde Liu $^{1}$, Bin Liang ${ }^{1,3, *}$ and Bo Yuan ${ }^{4}$
}

\begin{abstract}
This paper aims to propose a new robust stateestimation and fault-detection method by combining the unknown input observer (UIO) and the set-membership estimator (SME). It is known that both the SUIO and the SME can be used to estimate the states of a system. The former aims to obtain a particular value by actively decoupling the effect of unknown inputs, while the latter can obtain state-estimation sets by prediction and correction based on the set theory. Instead of particular state values, the latter can obtain state-estimation sets guaranteeing to contain system states (i.e., robust state estimation). In this paper, we propose to use the framework of the UIO to actively decouple part of unknown inputs and then further employ the set-membership estimation method to estimate state sets and detect faults. The objective of the proposed method is to reduce the conservatism of robust stateestimation sets by using the UIO to remove the contribution of part of unknown inputs to the sizes of state-estimation sets. At the end of this paper, a numerical example is used to illustrate the effectiveness and advantages of the proposed approach.
\end{abstract}

\section{INTRODUCTION}

For a system, it is not always possible to measure all the states. Regarding those unmeasurable states, we have to estimate them for the application purposes such as control design and fault diagnosis. As an important topic in the control field, the state estimation of a system has been attracting considerable attention [4], [7], [9].

In general, there are two ways to estimate system states. The first one is to use conventional observers such as Luenberger observers, unknown input observers, Kalman filters, adaptive observers to estimate a specific value for the system states at each time instant [8], [14], [17]. In some sense, it is difficult to assess the degree of approximation of those estimated values with respect to the corresponding accurate values. The second way is to obtain robust state estimation using the set-based approaches [1], [6]. A wellknown robust technique is the set-membership estimation [12], [15]. Instead of estimating a particular value, the setbased robust state-estimation techniques aim to obtain a state-estimation set that guarantees to confine the accurate value of states inside at each time instant. Since these techniques can give bounds to the states, we can assess the

\footnotetext{
${ }^{1}$ Center of Intelligent Control and Telescience, Graduate School at Shenzhen, Tsinghua University, 518055 Shenzhen, P.R.China.

${ }^{2}$ Institut de Robòtica i Informàtica Industrial (CSIC-UPC), Technical University of Catalonia (UPC), 08028 Barcelona, Spain.

${ }^{3}$ Tsinghua National Laboratory for Information Science and Technology, Tsinghua University, 100084 Beijing, P.R.China.

${ }^{4}$ Intelligent Computing Lab, Division of Informatics, Graduate School at Shenzhen, Tsinghua University, 518055 Shenzhen, P.R.China.

${ }^{*}$ Corresponding authors:Bin Liang and Xueqian Wang

E-mail:bliang@tsinghua.edu.cn, \{xu.feng,wang.xq\}@sz.tsinghua.edu.cn
}

accuracy of state estimation in an intuitive way. Moreover, the smaller the set is, the better the estimation is

In this paper, a new set-based state-estimation method is proposed to obtain better state-estimation sets, which integrates the framework of the UIO and the SME to implement the robust state estimation and fault detection. In order to reduce the conservatism of state-estimation sets, we have to reduce their sizes. The proposed idea is to take advantage of the UIO to decouple the effect of part of unknown inputs on the sizes of state-estimation sets [5], [10], [16]. In this way, the state-estimation problem of the system can be converted into that of a state-estimation-error system. Then, we use the SME to estimate the state of this transformed system whose state estimations are finally used to recover the corresponding robust state-estimation sets of the original system.

As a novel robust state-estimation approach, its most important feature is that the UIO framework is employed to remove the effect of some unknown inputs. Moreover, the design process does not require to satisfy the design conditions of UIOs in [5]. Instead, by using the set-theoretic unknown input observer (SUIO), it is quite easy to design it for the robust state-estimation and fault-detecttion purposes. Thus, a sufficient number of degrees of freedom can be obtained for designing the robust state estimator proposed in this paper. As a result, it is possible to obtain less conservative state-estimation sets by using this method, which is its most important contribution.

The remainder of this paper is organized as follows. Section II introduces the plant model and the principle of the set-membership-estimation approach. Section III presents the design of a robust state estimator proposed in this paper and its application to fault detection. Section IV illustrates the effectiveness of the proposed approach and compares it with the SME by using a numerical example. Finally, the paper is concluded in Section V with the future research directions.

\section{SYSTEM DESCRIPTION}

The system model, the UIO and the SME are introduced in this section, respectively.

\section{A. Plant Model}

The plant is assumed to be represented by the following linear discrete time-invariant model

$$
\begin{aligned}
x_{k+1} & =A x_{k}+B u_{k}+E \omega_{k}, \\
y_{k} & =C x_{k}+F \eta_{k},
\end{aligned}
$$

where $A \in \mathbb{R}^{n \times n}, B \in \mathbb{R}^{n \times p}, E \in \mathbb{R}^{n \times r}, C \in \mathbb{R}^{q \times n}$ and $F \in \mathbb{R}^{q \times s}$ are time-invariant matrices, $k$ denotes the $k$-th 
discrete time instant, $x_{k} \in \mathbb{R}^{n}$ and $y_{k} \in \mathbb{R}^{q}$ denote the state and output vectors, $u_{k} \in \mathbb{R}^{p}$ and $\omega_{k} \in \mathbb{R}^{r}$ represent the known and unknown inputs (i.e., process disturbances, modelling errors, linearizing errors, etc.), respectively, and $\eta_{k} \in \mathbb{R}^{s}$ represents the measurement noise vector.

Assumption 2.1: The pair $(A, C)$ is detectable.

Remark 1: Assumption 2.1 is used to guarantee the feasibility of designing a stable observer for the system.

In this paper, we focus on set-based robust state estimation of a monitored system. Thus, it is assumed that the measurement noise vector $\eta_{k}$ is bounded as shown in Assumption 2.2.

Assumption 2.2: The measurement noise vector $\eta_{k}$ is bounded by a set

$$
V=\left\{\eta \in \mathbb{R}^{s}:\left|\eta-\eta^{c}\right| \leq \bar{\eta}\right\},
$$

where $\eta^{c}$ and $\bar{\eta}$ are known and constant vectors.

In order to reduce computational complexity, zonotopes are chosen to implement set operations [1], [2]. Under Assumption 2.2, the set $V$ can be represented by

$$
V=\left\{\eta^{c}\right\} \oplus H_{\bar{\eta}} \mathbb{B}^{s},
$$

where $H_{\bar{\eta}}$ is a diagonal matrix whose diagonal elements are the components of $\bar{\eta}$ and $\oplus$ denotes the Minkowski sum.

\section{B. Set-membership Estimation}

It is assumed that the initial state $x_{0}$ is bounded by the initial zonotope $\hat{X}_{0}$ of the SME:

$$
x_{0} \in \hat{X}_{0} .
$$

Definition 2.1: Given the system (1) and a measured output $y_{k}$, the measurement-consistent set at time instant $k$ is defined as $X_{y_{k}}=\left\{x_{k} \in \mathbb{R}^{n}: C x_{k}-y_{k} \in-F V\right\}$.

Assumption 2.3: The vector $\omega_{k}$ is bounded by a set

$$
W=\left\{\omega \in \mathbb{R}^{r}:\left|\omega-\omega^{c}\right| \leq \bar{\omega}\right\},
$$

where $\omega^{c}$ and $\bar{\omega}$ are known and constant vectors.

Similarly, the set $W$ can be written into a zonotopic form. The set-membership estimation approach uses several steps to obtain robust state estimations by employing the state dynamics (1a) and output equation (1b), respectively (see [1] for more details).

- Prediction step: a zonotope $\bar{X}_{k}=A \hat{X}_{k-1} \oplus\left\{B u_{k}\right\} \oplus$ $E W$ is obtained to bound all possible values of states at time instant $k$, where $\hat{X}_{k-1}$ is a zonotope and $\hat{x}_{k-1} \in$ $\hat{X}_{k-1}$ holds at time instant $k-1$.

- Measurement step: the output $y_{k}$ is obtained and the current measurement-consistent set $X_{y_{k}}$ is computed.

- Correction step: the measurement-consistent set $X_{y_{k}}$ is used to correct the predicted set $\bar{X}_{k}$ and an intersection $X_{e k}=\bar{X}_{k} \cap X_{y_{k}}$ can be obtained.

- Zonotopic outer-approximation step: a zonotopic outerapproximation $\hat{X}_{k}$ to bound $X_{e k}$ can be constructed, which is used for the next-step prediction.

Remark 2: The set $X_{e k}$ is the exact state-estimation set at time instant $k$, which is consistent with the output $y_{k}$ and the state-estimation set $\hat{X}_{k-1}$. In general, it is difficult to obtain the exact state-estimation set $X_{e k}$ because of the complex geometric structure of sets. In order to reduce computational complexity, an outer approximation $\hat{X}_{k}$ with a simple geometric structure (zonotope) is used to bound the exact state-estimation set $X_{e k}$ for the propagation of the dynamics at next steps for $k>0$.

In order to implement the set-membership-estimation approach, Properties 2.1, 2.2 and 2.3 of zonotopes are used. Furthermore, in [11], an algorithm to compute a zonotopic outer-approximation of the intersection of a zonotope and a polytope is presented in Property 2.4.

Property 2.1: Given $X_{1}=g_{1} \oplus H_{1} \mathbb{B}^{r_{1}} \subset \mathbb{R}^{n}$ and $X_{2}=$ $g_{2} \oplus H_{2} \mathbb{B}^{r_{2}} \subset \mathbb{R}^{n}$, their Minkowski sum is $X_{1} \oplus X_{2}=$ $\left\{g_{1}+g_{2}\right\} \oplus\left[\begin{array}{ll}H_{1} & H_{2}\end{array}\right] \mathbb{B}^{r_{1}+r_{2}}$.

Property 2.2: Given $X=g \oplus H \mathbb{B}^{r} \subset \mathbb{R}^{n}$ and a suitable matrix $K, K X=K g \oplus K H \mathbb{B}^{r}$.

Property 2.3: ( [1]). Given a zonotope $X=g \oplus H \mathbb{B}^{r} \subset$ $\mathbb{R}^{n}$ and an integer $s$ (with $n<s<r$ ), denote by $\hat{H}$ the matrix resulting from the reordering of the columns of the matrix $H$ in decreasing Euclidean norm. $X \subseteq g \oplus$ $\left[\begin{array}{ll}\hat{H}_{T} & Q\end{array}\right] \mathbb{B}^{s}$ where $\hat{H}_{T}$ is obtained from the first $s-n$ columns of matrix $\hat{H}$ and $Q \in \mathbb{R}^{n \times n}$ is a diagonal matrix whose elements are $Q_{i i}=\sum_{j=s-n+1}^{r}\left|\hat{H}_{i j}\right|, i=1, \ldots, n$.

Property 2.4: ( [11]). Given a matrix $\Lambda \in \mathbb{R}^{n \times m}$, a zonotope $Z=g \oplus H \mathbb{B}^{r}$, and an $H$-polytope $P=\{x \in$ $\left.\mathbb{R}^{n}:|C x-d| \leq\left[\phi_{1}, \phi_{2}, \ldots, \phi_{m}\right]^{T}\right\}$, with $C \in \mathbb{R}^{m \times n}$, $d \in \mathbb{R}^{m}, \phi_{i} \in \mathbb{R}_{+}(i=1,2, \ldots, m)$, define a vector $\hat{g}(\Lambda)=$ $g+\Lambda(d-C g)$ and a matrix $\hat{H}(\Lambda)=\left[\left(\begin{array}{ll}I-\Lambda C) H & \Lambda \Phi\end{array}\right]\right.$, with a diagonal matrix $\Phi=\operatorname{diag}\left(\phi_{1}, \phi_{2}, \ldots, \phi_{m}\right)$. Then, a family of zonotopes (parameterized by the matrix $\Lambda$ ) that contains the intersection of the zonotope $Z$ and the polytope $P$ is obtained as $Z \cap P \subseteq \hat{Z}(\Lambda)=\hat{g} \oplus \hat{H} \mathbb{B}^{r+m}$.

\section{Robust State Estimation And Fault Detection}

This section proposes a method that combines the UIO with the SME to obtain robust state estimations and its application to fault detection.

\section{A. Design of SUIO}

The traditional SME is affected by all process disturbances and measurement noises. This implies that all the bounds of unknown inputs and measurement noises contribute to the size of state-estimation set. However, we always hope to reduce the size of state-estimation set as much as possible. Thus, if we can remove the effect of part of unknown inputs, it is possible to obtain less conservative state-estimation sets.

In this paper, the idea is to combine the SUIO with the SME to implement this objective, which uses the SUIO to remove the effect of part of unknown inputs and then utilizes the SME to further obtain robust state-estimation sets. Recently, in [16], a novel SUIO was proposed by the authors, where the proposed SUIO is used to overcome the existence conditions of traditional UIOs and simultaneously implement the active decoupling of part of unknown inputs. 
According to [5], for the system (1), a UIO can be designed as

$$
\begin{aligned}
z_{k+1} & =N z_{k}+T u_{k}+K y_{k}, \\
\hat{x}_{k} & =M z_{k}+H y_{k} \\
\hat{y}_{k} & =C \hat{x}_{k}
\end{aligned}
$$

where $z_{k} \in \mathbb{R}^{n}, \hat{x}_{k} \in \mathbb{R}^{n}$ and $\hat{y}_{k} \in \mathbb{R}^{q}$ are the state vector, estimated state and output vectors, $N \in \mathbb{R}^{n \times n}, T \in \mathbb{R}^{n \times p}$, $K \in \mathbb{R}^{n \times q}, M \in \mathbb{R}^{n \times n}$ and $H \in \mathbb{R}^{n \times q}$.

Theorem 3.1: The necessary and sufficient conditions for the observer (5) to exist for the system (1) are

- $\operatorname{rank}(C E)=\operatorname{rank}(E)$,

- $\left(C, A_{1}\right)$ is a detectable pair, where

$$
A_{1}=A-E\left[(C E)^{T} C E\right]^{-1}(C E)^{T} C A .
$$

Proof: The proof of Theorem 3.1 can be found in [5].

Under Theorem 3.1, it means that the observer (5) able to decouple all the unknown inputs contained in the vector $\omega_{k}$ can exist. However, in reality, a considerable number of systems cannot satisfy the conditions given in Theorem 3.1. In order to overcome this problem, we will design an SUIO to decouple part of unknown inputs included in $\omega_{k}$. In order to reach this aim, we rewrite $\omega_{k}$ into

$$
\omega_{k}=\left[\begin{array}{l}
\omega_{1, k} \\
\omega_{2, k}
\end{array}\right],
$$

where $\omega_{1, k} \in \mathbb{R}^{n_{a}}, \omega_{2, k} \in \mathbb{R}^{n_{p}}, n_{a}$ denotes the number of unknown inputs that we can design an SUIO to actively decouple, while $n_{p}$ is the remaining number of unknown inputs and is given by

$$
n_{p}=r-n_{a}
$$

Correspondingly, the matrix $E$ can be rewritten as

$$
E=\left[\begin{array}{ll}
E_{1} & E_{2}
\end{array}\right]
$$

where $E_{1} \in \mathbb{R}^{n \times n_{a}}$ and $E_{2} \in \mathbb{R}^{n \times n_{p}}$. In this case, the state dynamics (1a) can be further rewritten as

$$
x_{k+1}=A x_{k}+B u_{k}+E_{1} \omega_{1, k}+E_{2} \omega_{2, k} .
$$

Moreover, based on (1), (5) and (8), the state-estimationerror vector of the UIO is defined as

$$
e_{k}^{x}=x_{k}-\hat{x}_{k}
$$

with the dynamics

$$
\begin{aligned}
e_{k+1}^{x}= & \left(A-H C A-M K_{1} C\right) e_{k}^{x}+[(A-H C A \\
& \left.\left.-M K_{1} C\right) M-M N\right] z_{k}+[(A-H C A \\
& \left.\left.-M K_{1} C\right) H-M K_{2}\right] y_{k}+(B-M T-H C B) u_{k} \\
& +\left(E_{1}-H C E_{1}\right) \omega_{1, k}+\left(E_{2}-H C E_{2}\right) \omega_{2, k} \\
& -H F \eta_{k+1}-M K_{1} F \eta_{k},
\end{aligned}
$$

where

$$
K=K_{1}+K_{2}
$$

Since $\omega_{1, k}$ denotes the unknown inputs that can be actively decoupled by an SUIO, the parametric matrices of (5) can always be designed to satisfy

$$
\begin{aligned}
E_{1}-H C E_{1} & =\mathbf{0}, \\
\left(A-H C A-M K_{1} C\right) M-M N & =\mathbf{0} .
\end{aligned}
$$

According to (10) and (12), the dynamics of the stateestimation-error can be equivalently reduced into

$$
\begin{aligned}
e_{k+1}^{x}= & \left(A-H C A-M K_{1} C\right) e_{k}^{x}+[(A-H C A \\
& \left.\left.-M K_{1} C\right) H-M K_{2}\right] y_{k}+(B-M T \\
& -H C B) u_{k}+\left(E_{2}-H C E_{2}\right) \omega_{2, k} \\
& -H F \eta_{k+1}-M K_{1} F \eta_{k},
\end{aligned}
$$

where it can be seen that $\omega_{1, k}$ is decoupled by the SUIO.

Similarly, we can define and compute the corresponding output-estimation-error vector as

$$
e_{k}^{y}=y_{k}-\hat{y}_{k}=C e_{k}^{x}+F \eta_{k} .
$$

By using (5), the system (1) can be mirrored into a different but equivalent dynamics based on (13) and (14):

$$
\begin{aligned}
e_{k+1}^{x} & =A^{e} e_{k}^{x}+B^{e} u_{k}^{e}+E^{e} v_{k} \\
e_{k}^{y} & =C e_{k}^{x}+F \eta_{k}
\end{aligned}
$$

with

$$
\begin{aligned}
& A^{e}=\left[A-H C A-M K_{1} C\right], \\
& B^{e}=\left[\left(A-H C A-M K_{1} C\right) H-M K_{2}\right. \\
& B-M T-H C B] \text {, } \\
& E^{e}=\left[\begin{array}{lll}
E_{2}-H C E_{2} & -H F & -M K_{1} F
\end{array}\right], \\
& u_{k}^{e}=\left[\begin{array}{ll}
y_{k}^{T} & u_{k}^{T}
\end{array}\right]^{T} \text {, } \\
& v_{k}=\left[\begin{array}{lll}
\omega_{2, k}^{T} & \eta_{k+1}^{T} & \eta_{k}^{T}
\end{array}\right]^{T},
\end{aligned}
$$

where the superscript $T$ denotes the transpose of vector.

In this paper, we propose to transform the robust stateestimation problem of the system (1) into solving that of the system (15).

\section{B. Robust State Estimation}

According to Section II-B, Assumption 2.3 is needed for the implementation of the traditional SME. However, in this paper, instead of Assumption 2.3, we only need that the unknown input vector $\omega_{2, k}$ is bounded.

Assumption 3.1: The unknown input vector $\omega_{2, k}$ is bounded by a set

$$
W_{2}=\left\{\omega_{2} \in \mathbb{R}^{n_{p}}:\left|\omega_{2}-\omega_{2}^{c}\right| \leq \bar{\omega}_{2}\right\},
$$

where $\omega_{2}^{c}$ and $\bar{\omega}_{2}$ are constant and known vectors.

Remark 3: Since the SUIO can decouple the effect of the unknown input vector $\omega_{1, k}$, an advantage of the proposed robust state-estimation method over the traditional SME is that only part of the unknown inputs are needed to be bounded (i.e., $\omega_{2, k} \in W_{2}$ ).

Furthermore, considering that $\omega_{2, k}, \eta_{k+1}$ and $\eta_{k}$ are bounded, $v_{k}$ is also bounded and its set can be computed 
by using the sets $W_{2}$ and $V$. In this paper, we denote the set of $v_{k}$ as $S$, that is

$$
v_{k} \in S
$$

Under the assumption given in (3), the initial value of the dynamics (15) should satisfy

$$
e_{0}^{x} \in \hat{E}_{0}^{x},
$$

with

$$
\begin{aligned}
e_{0}^{x} & =x_{0}-\hat{x}_{0}, \\
\hat{E}_{0}^{x} & =x_{0} \oplus\left(-\hat{X}_{0}\right),
\end{aligned}
$$

where $\hat{X}_{0}$ is designed such that $\hat{x}_{0} \in \hat{X}_{0}$ holds and $\hat{E}_{0}^{x}$ is used to denote the initial set of $e_{0}^{x}$.

Remark 4: The energy of a physical system is always limited. This implies that it is always possible to design a pair $\left(\hat{x}_{0}, \hat{X}_{0}\right)$ to guarantee $e_{0}^{x} \in \hat{E}_{0}^{x}$.

According to (16) and (17), we can apply the framework of the SME into the dynamics (15). Particularly, a prediction set of $e_{k}^{x}$ can be obtained by propagating (15a) as

$$
\bar{E}_{k}^{x}=A^{e} \hat{E}_{k-1}^{x} \oplus\left\{B^{e} u_{k}^{e}\right\} \oplus E^{e} S, k>0 .
$$

At the same time instant, $e_{k}^{y}$ can be computed by using (1b) and (5c). Thus, with $e_{k}^{y}$ and (15b), we can further compute a set $E_{k}^{y}$ of $e_{k}^{x}$ consistent to $e_{k}^{y}$, where $E_{k}^{y}$ is computed from

$$
C e_{k}^{x}-e_{k}^{y} \in-F V \text {. }
$$

According to Section II-B, the robust state-estimation set of $e_{k}^{x}$ can be obtained by intersecting the prediction set $\bar{E}_{k}^{x}$ and the consistent set $E_{k}^{y}$ as

$$
E_{e k}^{x}=\bar{E}_{k}^{x} \cap E_{k}^{y},
$$

where it can be guaranteed that

$$
e_{k}^{x} \in E_{e k}^{x} .
$$

Similar to the traditional SME, in order to predict the set $\bar{E}_{k}^{x}$ given in (18) in real time, we have to construct an outerapproximate zonotope $\hat{E}_{k}^{x}$ to bound the set $E_{e k}^{x}$ :

$$
E_{e k}^{x} \subseteq \hat{E}_{k}^{x}
$$

In this case, we will be able to repetitively estimate robust state-estimation sets of $e_{k}^{x}$ for all $k>0$.

As a result, the main results of this paper on robust state estimations are summarized in the following proposition.

Proposition 3.1: Under Assumptions 2.1, 2.2 and 3.1, Theorem 3.1, and (3) and (17), the robust state-estimation set of the system (1) at time instant $k$ can be obtained by using (5), (15), (18), (19) and (20) as

$$
X_{e k}=\hat{x}_{k} \oplus E_{e k}^{x} .
$$

Proof: According to (21), we can have $e_{k}^{x}=x_{k}-\hat{x}_{k} \in$ $E_{e k}^{x}$. In this way, it can be further obtained that $x_{k} \in \hat{x}_{k} \oplus$ $E_{e k}^{x}$ holds. That is, $x_{k} \in X_{e_{k}}$.

It can be seen that the proposed robust state-estimation approach is based on the combination of the UIO framework and the SME. However, we should emphasize that this approach only uses the UIO framework. The reason is that, different form the design of SUIOs to require that all the parametric matrices of $z_{k}, y_{k}, u_{k}$ and $\omega_{1, k}$ in (10) must be zero matrices, that is,

$$
\begin{aligned}
E_{1}-H C E_{1} & =\mathbf{0}, \\
B-M T-H C B & =\mathbf{0}, \\
\left(A-H C A-M K_{1} C\right) M-M N & =\mathbf{0}, \\
\left(A-H C A-M K_{1} C\right) H-M K_{2} & =\mathbf{0},
\end{aligned}
$$

which are used to guarantee that the state-estimation-error vector will converge to zero. As a comparison, the proposed method only requires to satisfy (24a) and (24c), which implies less conservative design conditions with respect to the SUIO and more freedom to design the parametric matrices of (5) to achieve some extra performance. Consequently, it results in that the vector $e_{k}^{x}$ does not converge to $\mathbf{0}$ as $k$ tends to $\infty$ (i.e., (5) is not a real observer (UIO/SUIO) for the system (1)). Thus, in this proposed robust state-estimation approach, we only have one set-based state estimator, which is composed of (5), (15), (18), (19), (20), (22) and (23).

Note that, under the framework of the proposed robust state estimator, it is possible to relax the design conditions by removing (12b). Moreover, if the design freedom of the proposed observer allows, we can also design the parametric matrix $M K_{1} F$ of $\eta_{k}$ to be zero to further reduce the state estimation and fault detection conservatism. However, this also requires a degree of extension of the current results.

\section{Application to Fault Detection}

An important application of state-estimation techniques is in the field of the model-based fault detection. As a well known fault-detection method, the model-based method uses state/output estimations of a system to compare with its real signals for the generation of residual signals [3]. Then, the residual signals are used for the fault-detection purposes by using distinct approaches.

However, the set-based state-estimation techniques are used for robust fault detection in a way different from the general residual generation and analysis, which is based on the consistency testing between the predicted state sets from the state dynamics and the output-consistent sets of states computed by using the output equation (see [1], [13]). A similar fault-detection criterion to that in [13] using the proposed state estimator is given in Proposition 3.2.

Proposition 3.2: Considering the plant (1) and the state estimator described by (5), (15), (18), (19), (20), (22) and (23), if $E_{e k}^{x}$ or $X_{e k}$ is empty, it can be guaranteed that the plant has already become faulty at time instant $k$.

Proof: The emptiness of the set $E_{e k}^{x}$ or $X_{e k}$ implies that there are no state values in the predicted set $\bar{E}_{k}^{x}$ that can explain the current value $e_{k}^{y}$ computed by using (15b) from the real measured output vector $y_{k}$. Moreover, since the sets of process disturbances and measurement noises have been taken into account when computing those sets, it can be guaranteed that the plant has become faulty. 
Thus, by using the fault-detection criterion in Proposition 3.2, we can further use the proposed state-estimation approach to robustly detect faults.

\section{ILLUSTRATIVE EXAMPLE}

In this paper, the effectiveness of the proposed method is illustrated by a numerical example modeled as (1) with

$$
\begin{aligned}
A & =\left[\begin{array}{cc}
0.75 & 0 \\
0 & 0.7
\end{array}\right], B=\left[\begin{array}{cc}
0.5 & 0 \\
0 & 0.8
\end{array}\right], C=\left[\begin{array}{cc}
0.9 & 0 \\
0 & 0.8
\end{array}\right], \\
E & =\left[\begin{array}{ccc}
0.2 & 0.1 & 0.05 \\
0.1 & 0.25 & 0.2
\end{array}\right], F=\left[\begin{array}{ll}
1 & 0 \\
0 & 1
\end{array}\right] .
\end{aligned}
$$

There are three unknown inputs considered in this example. We use the proposed method to decouple the first unknown input. Thus, we can obtain

$$
E_{1}=\left[\begin{array}{l}
0.2 \\
0.1
\end{array}\right], E_{2}=\left[\begin{array}{cc}
0.1 & 0.05 \\
0.25 & 0.2
\end{array}\right]
$$

The parametric matrices of (5) are designed as

$$
\begin{aligned}
N & =\left[\begin{array}{cc}
0.5549 & -0.1474 \\
-0.8233 & -0.5982
\end{array}\right], T=\left[\begin{array}{ll}
0.5 & 0.6 \\
0.4 & 0.3
\end{array}\right], \\
K_{1} & =\left[\begin{array}{ll}
0.1597 & 0.4253 \\
0.7168 & 0.4814
\end{array}\right], K_{2}=\left[\begin{array}{ll}
0.65 & 0.43 \\
0.12 & 0.68
\end{array}\right], \\
K & =\left[\begin{array}{ll}
0.8097 & 0.8553 \\
0.8368 & 1.1614
\end{array}\right], M=\left[\begin{array}{ll}
0.3 & 0.6 \\
0.4 & 1.1
\end{array}\right], \\
H & =\left[\begin{array}{ll}
0.9278 & 0.4124 \\
0.4639 & 0.2062
\end{array}\right] .
\end{aligned}
$$

\section{A. Robust State Estimation}

The zonotopic sets of part of process disturbances and measurement noises are given as

$$
\begin{aligned}
W^{2} & =\left[\begin{array}{l}
0 \\
0
\end{array}\right] \oplus\left[\begin{array}{cc}
0.025 & 0 \\
0 & 0.02
\end{array}\right] \mathbb{B}^{2}, \\
V & =\left[\begin{array}{l}
0 \\
0
\end{array}\right] \oplus\left[\begin{array}{cc}
0.001 & 0 \\
0 & 0.001
\end{array}\right] \mathbb{B}^{2} .
\end{aligned}
$$

According to (15), (18), (19), (20), (22) and (23), we can use the proposed method to estimate robust state-estimation sets for the example. Moreover, in order to show the effectiveness of the approach, a comparison between this approach and the standard SME is carried out.

However, in order to use the standard SME approach, we have to assume that all process disturbances are bounded (i.e., $\omega_{1, k}$ should be bounded as well) as follows:

$$
W^{1}=[0] \oplus[0.2] \mathbb{B}^{1} .
$$

Under the condition that all the process disturbances are bounded, by using the SME introduced in Section II-B, we can also compute the set-based robust state estimations of the considered system. The results of the two state-estimation approaches are shown in Figure 1, where the time span of simulation is 100 time instants.

Remark 5: In Figure 1, $x_{k}(1)$ and $x_{k}(2)$ are the first and second components of the real states, respectively. $X_{e k}^{1}(1)$ and $X_{e k}^{1}(2)$ are the first and second components of the stateestimation sets of the proposed method, respectively. $X_{e k}^{2}(1)$
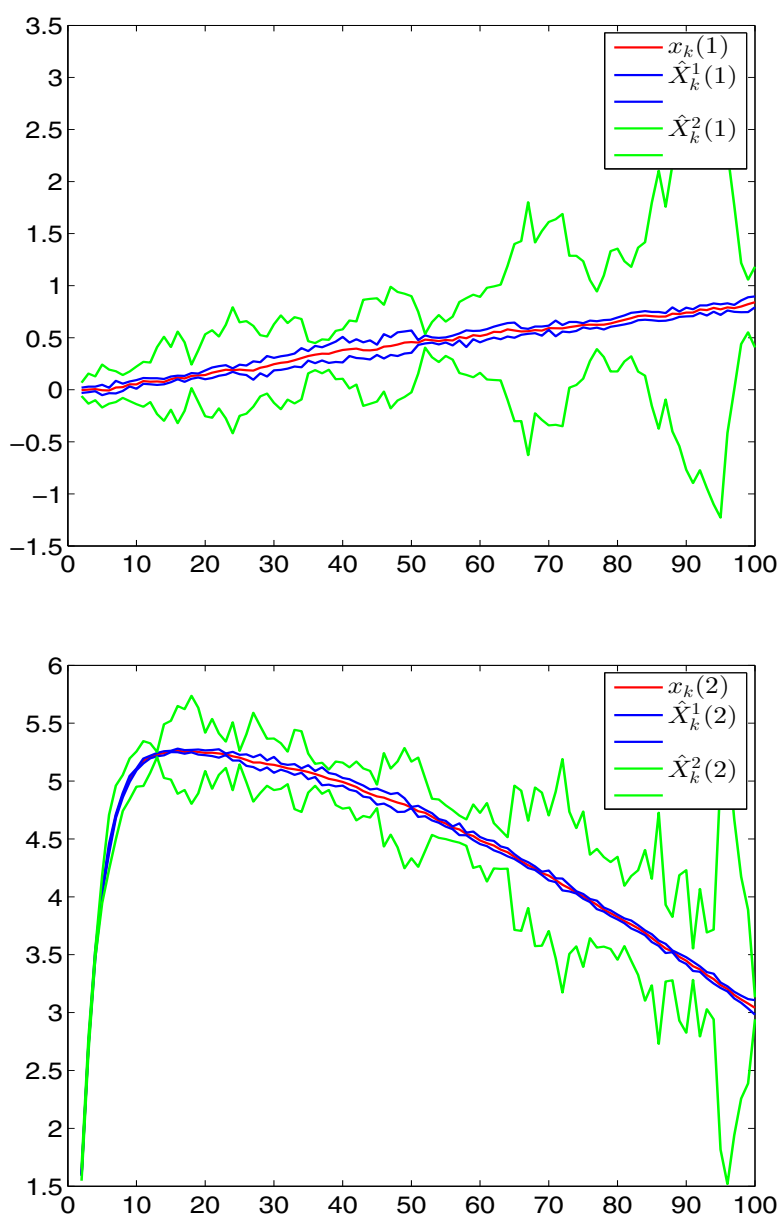

Fig. 1. Set-based state estimations

and $X_{e k}^{2}(2)$ are the first and second components of the stateestimation sets of the standard SME, respectively.

According to the simulation results, it can be observed that the proposed approach can obtain less conservative stateestimation sets than the standard set-membership-estimation approach for the application in this example.

Remark 6: Without loss of generality, the plots in Figure 1 are based on the interval hull of zonotopic outerapproximation $\hat{X}_{k}$ of the state-estimation set $X_{e k}$ at each time instant for simplicity.

\section{B. Robust Fault Detection}

In order to show the application of the proposed state estimator to fault detection, a fault scenario is defined. That is from the time instant $k=1$ to $k=50$, the system operates in the healthy situation, while at $k=51$, an actuatorparametric fault modeled by a fault matrix $F_{a}$ occurs, where the actuator-fault situation is modeled as

$$
x_{k+1}=A x_{k}+B F_{a} u_{k}+E w_{k} .
$$

In the considered scenario, the fault matrix is set to be

$$
F_{a}=\left[\begin{array}{cc}
1 & 0 \\
0 & 0.95
\end{array}\right] \text {. }
$$


We present the fault detection results in Figure 2, where the first and second plots corresponding to the proposed estimator-based and the standard SME-based methods, respectively. It can be seen that the former can detect the fault at $k_{d_{1}}=52$ while the latter detects the fault three time instants later (i.e., $k_{d_{2}}=55$ ), where $k_{d_{1}}$ and $k_{d_{2}}$ denotes the fault detection time instants corresponding to the proposed estimator-based and the SME-based methods, respectively. As a result, it is shown in Figure 2 that the proposed estimator-based method is more sensitive to the considered fault in this example.
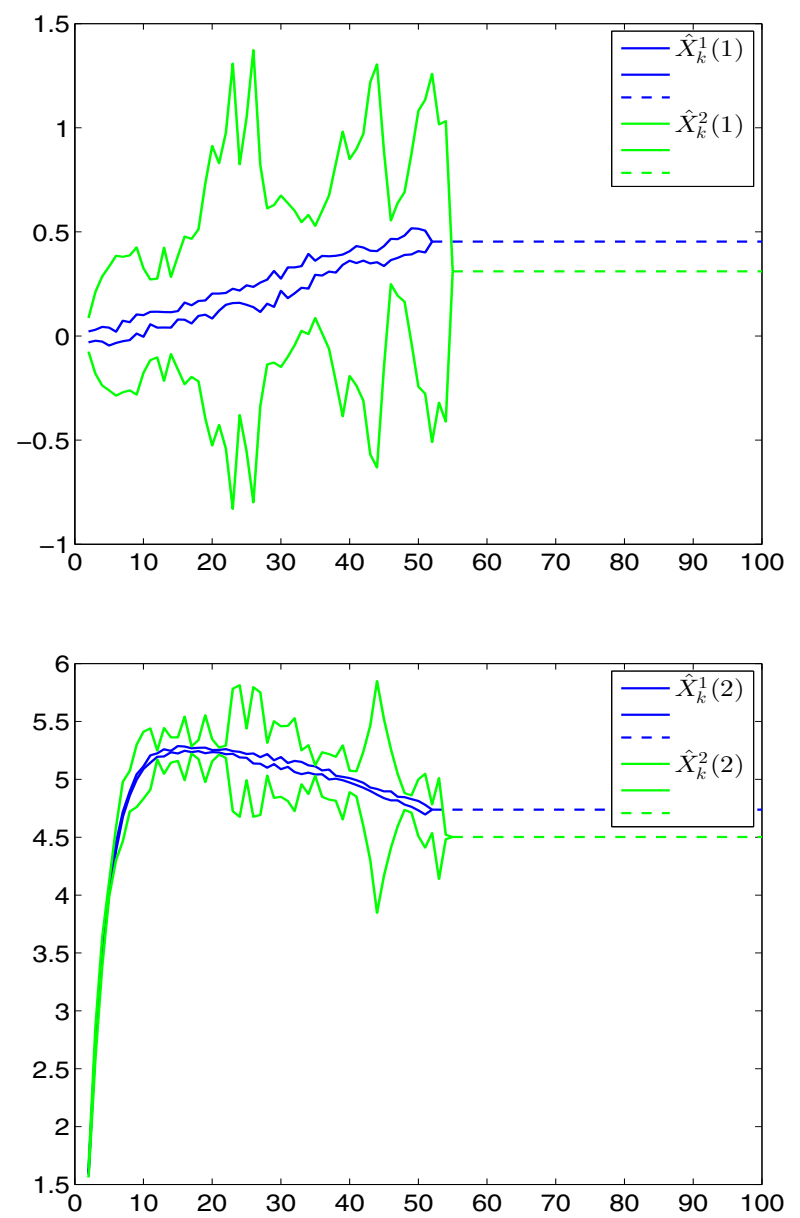

Fig. 2. Fault-detection results

Remark 7: In Figure 2, the dashed lines mean that the state-estimation sets are considered as empty sets after FD.

\section{Conclusions}

This paper proposed a set-based robust state estimator integrating the SUIO and the SME. Comparing with the existing set-based approaches, this robust state estimator can reduce the effect of part of unknown inputs such as process disturbances and linearizing errors. In this case, it is possible for us to obtain less conservative state-estimation sets. Moreover, the design of the proposed estimator can overcome the design conditions of the standard UIO. Additionally, since the design conditions (12) are much weaker than those in (24), it means that we can get more freedom to achieve better performance for the purposes of state estimation and fault detection. In the future research, an important direction is to further extend the proposed approach into the field of fault isolation, fault estimation and fault-tolerant control.

\section{ACKNOWLEDGEMENTS}

This work has been supported by the Natural Science Foundation of Guangdong (China) (No.2015A030313881), the Shenzhen Key Lab of Space Robotic Technology and Telescience (China), the Spanish Government (MINECO) through the project CICYT ECOCIS (ref. DPI2013-48243C2-1-R), and MINECO and FEDER through the project CICYT HARCRICS (ref. DPI2014-58104-R).

\section{REFERENCES}

[1] T. Alamo, J.M. Bravo, and E.F. Camacho. Guaranteed state estimation by zonotopes. Automatica, 41(6):1035-1043, 2005.

[2] F. Blanchini and S. Miani. Set-theoretic Methods in Control. Birkhäuser Boston, 2008.

[3] M. Blanke, M. Kinnaert, J. Lunze, and M. Staroswiecki. Diagnosis and Fault-Tolerant Control. Springer-Verlag, Berlin, Germany, 2006.

[4] M. Bolajraf and M. A. Rami. A robust estimation approach for uncertain systems with perturbed measurements. International Journal of Robust and Nonlinear Control, 26(4), 2016.

[5] J. Chen, R.J. Patton, and Hong-Yue Zhang. Design of unknown input observers and robust fault detection filters. International Journal of Control, 63(1):85-105, 1996.

[6] C. Combastel. A state bounding observer based on zonotopes. In European Control Conference, Cambridge, UK, 2003.

[7] C. Combastel, V. Puig, T. Rässi, and T. Alamo. Set-membership methods applied to FDI and FTC. International Journal of Adaptive Control and Signal Processing, 30(2):150-153, 2016.

[8] S. S. Delshad, A. Johansson, M. Darouach, and T. Gustafsson. Robust state estimation and unknown inputs reconstruction for a class of nonlinear systems: Multiobjective approach. Automatica, 64:1-7, 2016.

[9] J. George. A robust estimator for stochastic systems under unknown persistent excitation. Automatica, 63:156-161, 2016.

[10] S. Hui and S. H. Żak. Observer design for systems with unknown inputs. International Journal of Applied Mathematics and Computer Science, 15(4):431-446, 2005.

[11] V.T.H. Le, C.N. Stoica, T. Alamo, E.F. Camacho, and D. Dumur. Zonotope-based set-membership estimation for multi-output uncertain systems. In Proceedings of 2013 IEEE international Symposium on Intelligent Control (ISIC), Part of 2013 IEEE Multi-Conference on Systems and Control, Hyderabad, India, August 2013.

[12] Y. Liu, Y. Zhao, and F. Wu. Ellipsoidal state-bounding-based setmembership estimation for linear system with unknown-but-bounded disturbances. IET Control Theory and Applications, 10:431-442(11), February 2016.

[13] P. Rosa, C. Silvestre, J.S. Shamma, and M. Athans. Fault detection and isolation of ltv systems using set-valued observers. In Proceedings of the 49th IEEE Conference on Decision and Control, Hilton Atlanta Hotel, Atlanta, GA, USA, December 15-17 2010.

[14] K. Vijayaraghavan and A. Valibeygi. Adaptive nonlinear observer for state and unknown parameter estimation in noisy systems. International Journal of Control, 89(1):38-54, 2016.

[15] W. Wang, X. Liu, Y. Li, and Y. Liu. Set-membership filtering for genetic regulatory networks with missing values. Neurocomputing, 175, Part A:466 - 472, 2016.

[16] F. Xu, J.B. Tan, X.Q. Wang, V. Puig, B. Liang, and B. Yuan. A novel design of unknown input observers using set-theoretic methods for robust fault detection. In Accepted to the 2016 American Control Conference, Boston, United States, July 2016.

[17] Q. Yang and Y. Liu. Adaptive state estimation of multi-input and multi-output non-linear systems with general uncertainties both in the state and output equations. IET Control Theory and Applications, 10:354-362(8), February 2016. 